\title{
An Inequality for Hilbert-Schmidt Norm
}

\section{Huzihiro Araki and Shigeru Yamagami}

Research Institute for Mathematical Sciences, Kyoto University, Kyoto 606, Japan

\begin{abstract}
For the absolute value $|C|=\left(C^{*} C\right)^{1 / 2}$ and the Hilbert-Schmidt norm $\|C\|_{\mathrm{HS}}=\left(\operatorname{tr} C^{*} C\right)^{1 / 2}$ of an operator $C$, the following inequality is proved for any bounded linear operators $A$ and $B$ on a Hilbert space
\end{abstract}

$$
\||A|-|B|\|_{\mathrm{HS}} \leqq 2^{1 / 2}\|A-B\|_{\mathrm{HS}} \text {. }
$$

The corresponding inequality for two normal states $\varphi$ and $\psi$ of a von Neumann algebra $\mathbf{M}$ is also proved in the following form:

$$
d(\varphi, \psi) \leqq\|\xi(\varphi)-\xi(\psi)\| \leqq 2^{1 / 2} d(\varphi, \psi) .
$$

Here $\xi(\chi)$ denotes the unique vector representative of a state $\chi$ in a natural positive cone $\mathscr{P}^{\natural}$ for $\mathbf{M}$, and $d(\varphi, \psi)$ denotes the Bures distance defined as the infimum (which is also the minimum) of the distance of vector representatives of $\varphi$ and $\psi$. In particular,

$$
\left\|\xi\left(\varphi_{1}\right)-\xi\left(\varphi_{2}\right)\right\| \leqq 2^{1 / 2}\left\|\xi_{1}-\xi_{2}\right\|
$$

for any vector representatives $\xi_{j}$ of $\varphi_{j}, j=1,2$.

\section{Main Results}

In a study of quasi-equivalence of quasifree states of canonical commutation relations, we have encountered the following inequality, which seems to have an independent interest and hence we present it here as an independent article.

Theorem 1. For any two bounded linear operators $A$ and $B$ on a Hilbert space $\mathbf{H}$,

$$
\||A|-|B|\|_{\mathrm{HS}} \leqq 2^{1 / 2}\|A-B\|_{\mathrm{HS}} .
$$

Remark. The coefficient $2^{1 / 2}$ is the best possible for a general $A$ and $B$. If $A$ and $B$ are restricted to be selfadjoint, then the best coefficient is 1 instead of $2^{1 / 2}$. (Lemma 5.2, [1].) 
The above theorem can be reformulated in the context of (non-commutative) $L_{2}$-space over a von Neumann algebra $\mathbf{M}$. A vector $\xi$ in a representation space $\mathbf{H}$ of $\mathbf{M}$ is called a vector representative of a state $\varphi$ of $\mathbf{M}$ if

$$
\varphi(A)=(A \xi, \xi)
$$

holds for all $A \in \mathbf{M}$. [We have omitted the distinction between the representative operator, say $\pi(A)$, and $A$ itself.] In a standard representation space of $\mathbf{M}$ obtained from a normal semifinite faithful weight $\varphi_{0}$ of $\mathbf{M}$ by GelfandNaimark-Segal-(GNS) construction, the closure of vectors $\Delta^{1 / 4} \eta(x)$ with $x \in \mathbf{M}$, $x \geqq 0, \varphi_{0}\left(x^{2}\right)<\infty$, is called the natural positive cone and denoted by $\mathscr{P}^{\natural}$, where $\eta(x)$ is the GNS-representative vector of $x \in \mathbf{M}$. Any normal state $\varphi$ of $\mathbf{M}$ has a unique vector representative $\xi(\varphi)$ belonging to $\mathscr{P}^{\natural}$. (For example, Theorem 6 , [2].)

The infimum of the distance $\left\|\xi_{1}-\xi_{2}\right\|$ for vector representatives $\xi_{j}$ of normal states $\varphi_{j}(j=1,2)$ of $\mathbf{M}$ (infimum taken over all possible representation spaces of $\mathbf{M}$ as well as over all possible representative vectors in the space) is called the Bures distance of $\varphi_{1}$ and $\varphi_{2}$ and denoted by $d\left(\varphi_{1}, \varphi_{2}\right)$.

Theorem 2. For any two normal states $\varphi_{1}$ and $\varphi_{2}$ of $\mathbf{M}$,

$$
d\left(\varphi_{1}, \varphi_{2}\right) \leqq\left\|\xi\left(\varphi_{1}\right)-\xi\left(\varphi_{2}\right)\right\| \leqq 2^{1 / 2} d\left(\varphi_{1}, \varphi_{2}\right) .
$$

\section{Proof of Theorem 1 for Hilbert-Schmidt Class Operators}

In this section, we prove (1.1) for $A$ and $B$ in the Hilbert-Schmidt class. This result will be used afterwards for the proof of the general case.

For two operators $R$ and $S$ in the Hilbert-Schmidt class, we obtain the following by Schwartz inequality:

$$
2|\operatorname{tr}(S R)| \leqq 2\left(\operatorname{tr} S S^{*}\right)^{1 / 2}\left(\operatorname{tr} R^{*} R\right)^{1 / 2} \leqq \operatorname{tr} S^{*} S+\operatorname{tr} R^{*} R .
$$

By applying this twice, we obtain the following inequality for $X$ and $Y$ in the Hilbert-Schmidt class and a bounded linear operator $Q$ satisfying $X \geqq 0, Y \geqq 0$ and $\|Q\| \leqq 1$

$$
\begin{aligned}
4|\operatorname{tr} Q X Y| & =4\left|\operatorname{tr}\left(Y^{1 / 2} Q X^{1 / 2}\right)\left(X^{1 / 2} Y^{1 / 2}\right)\right| \\
& \leqq 2\left(\operatorname{tr} X^{1 / 2} Q^{*} Y Q X^{1 / 2}+\operatorname{tr} Y^{1 / 2} X Y^{1 / 2}\right) \\
& =2 \operatorname{tr} Y Q X Q^{*}+2 \operatorname{tr} X Y \\
& \leqq \operatorname{tr} Q^{*} Y^{2} Q+\operatorname{tr} Q X^{2} Q^{*}+2 \operatorname{tr} X Y \\
& \leqq \operatorname{tr}\left(X^{2}+Y^{2}+X Y+Y X\right)
\end{aligned}
$$

Let $A=U|A|$ and $B=V|B|$ be the polar decompositions of $A$ and $B$. By using the above inequality for $X=|A|, \mathrm{Y}=|B|$ and $Q=V^{*} U$, we obtain

$$
\begin{aligned}
2\|A-B\|_{\mathrm{HS}}^{2} & =2\left(\operatorname{tr}\left(|A|^{2}+|B|^{2}\right)-2 \operatorname{Re} \operatorname{tr}|B| V^{*} U|A|\right) \\
& \geqq 2 \operatorname{tr}\left(|A|^{2}+|B|^{2}\right)-\operatorname{tr}\left(|A|^{2}+|B|^{2}+|A||B|+|B||A|\right) \\
& =\operatorname{tr}(|A|-|B|)^{2}=\||A|-|B|\|_{\mathrm{HS}}^{2} .
\end{aligned}
$$




\section{Proof of Theorem 2}

This is almost exactly the same as the preceding proof. Let $Q \in \mathbf{M}$ and $\|Q\| \leqq 1$. Let $s(\varphi)$ denote the support projection of a state $\varphi$ of $\mathbf{M}, j(x)=J x J, J$ be the modular conjugation associated with the positive natural cone $\mathscr{P}^{\natural}$ and $\Delta_{\psi \varphi}$ be the relative modular operator of two states $\varphi$ and $\psi$ defined by (for example)

$$
\Delta_{\psi \varphi}^{1 / 2}(x \xi(\varphi)+(1-j(s(\varphi))) \psi)=J_{S}(\varphi) x^{*} \xi(\psi),
$$

where $\xi(\cdot)$ denotes the vector representative of a state in $\mathscr{P}^{\natural}, x$ runs over $\mathbf{M}, \Psi$ runs over the space $\mathbf{H}$, and $\Delta_{\psi \varphi}^{1 / 2}$ is the closure of the operator defined by (3.1) and is positive selfadjoint with its square defining $\Delta_{\psi \varphi}$.

Since $\overline{M \xi(\varphi)}=j(s(\varphi)) H$ and $J \Psi=\Psi$ for any $\Psi \in \mathscr{P}^{\natural}$, we obtain

$$
\begin{aligned}
4|(Q \xi(\varphi), \xi(\psi))| & =4|(j(s(\varphi)) Q \xi(\varphi), J \xi(\psi))| \\
& \left.=4\left|\left(Q \xi(\varphi), J_{S}(\varphi) \xi(\psi)\right)\right| \quad \text { (antiunitarity of } J \text { and } J^{2}=1\right) \\
& =4\left|\left(Q \xi(\varphi), \Delta_{\psi \varphi}^{1 / 2} \xi(\varphi)\right)\right| \quad(\text { by }(3.1)) \\
& =4\left|\left(\Delta_{\psi \varphi}^{1 / 4} Q \xi(\varphi), \Delta_{\psi \varphi}^{1 / 4} \xi(\varphi)\right)\right| \\
& \leqq 2\left(\left\|\Delta_{\psi \varphi}^{1 / 4} Q \xi(\varphi)\right\|^{2}+\left\|\Delta_{\psi \varphi}^{1 / 4} \xi(\varphi)\right\|^{2}\right) \\
& =2\left\{\left(\Delta_{\psi \varphi}^{1 / 2} Q \xi(\varphi), Q \xi(\varphi)\right)+\left(\Delta_{\psi \varphi}^{1 / 2} \xi(\varphi), \xi(\varphi)\right)\right\} \\
& \left.=2\left\{J_{S}(\varphi) Q^{*} \xi(\psi), Q \xi(\varphi)\right)+\left(J_{S}(\varphi) \xi(\psi), \xi(\varphi)\right)\right\} \\
& \leqq\left\|J_{S}(\varphi) Q^{*} \xi(\psi)\right\|^{2}+\|Q \xi(\varphi)\|^{2}+2(j(s(\varphi)) \xi(\psi), \xi(\varphi)) \\
& \leqq\left\|J_{S}(\varphi) Q^{*}\right\|^{2}\|\xi(\psi)\|^{2}+\|Q\|^{2}\|\xi(\varphi)\|^{2}+2(\xi(\psi), \xi(\varphi)) \\
& \leqq\|\xi(\psi)\|^{2}+\|\xi(\varphi)\|^{2}+(\xi(\psi), \xi(\varphi))+(\xi(\varphi), \xi(\psi)),
\end{aligned}
$$

where we have used $(\xi(\varphi), \xi(\psi))=(\xi(\psi), \xi(\varphi))(\geqq 0)$ in the last line.

If $\xi_{1}$ and $\xi_{2}$ are vector representatives of $\varphi_{1}$ and $\varphi_{2}$, then there exist partial unitaries $u_{j}$ in $\mathbf{M}^{\prime}$ such that $\xi_{j}=u_{j} \xi\left(\varphi_{j}\right)(j=1,2)$. We obtain from the above inequality for $Q=j\left(u_{1}^{*} u_{2}\right)^{*} \in \mathbf{M}$ the following:

$$
\begin{aligned}
\left(\xi_{2}, \xi_{1}\right) & =\left(u_{1}^{*} u_{2} \xi\left(\varphi_{2}\right), \xi\left(\varphi_{1}\right)\right)=\left(J \xi\left(\varphi_{1}\right), J u_{1}^{*} u_{2} \xi\left(\varphi_{2}\right)\right) \\
& =\left(\xi\left(\varphi_{1}\right), Q^{*} \xi\left(\varphi_{2}\right)\right)
\end{aligned}
$$

[by $J \xi\left(\varphi_{j}\right)=\xi\left(\varphi_{j}\right)$ and $J^{2}=1$ ]. Noting that $\left\|\xi_{j}\right\|^{2}=\left\|\xi\left(\varphi_{j}\right)\right\|^{2}=\varphi_{j}(1)$, we obtain

$$
\begin{aligned}
2\left\|\xi_{1}-\xi_{2}\right\|^{2} & =2\left(\left\|\xi_{1}\right\|^{2}+\left\|\xi_{2}\right\|^{2}-2 \operatorname{Re}\left(\xi_{2}, \xi_{1}\right)\right) \\
& \geqq 2\left(\left\|\xi\left(\varphi_{1}\right)\right\|^{2}+\left\|\xi\left(\varphi_{2}\right)\right\|^{2}-2\left|\left(\xi_{2}, \xi_{1}\right)\right|\right) \\
& \geqq\left\|\xi\left(\varphi_{1}\right)-\xi\left(\varphi_{2}\right)\right\|^{2} .
\end{aligned}
$$

\section{Proof of a Weaker Version of Theorem 1}

For an approximation argument, we need the following:

Proposition. If $\lim _{n \rightarrow \infty}\left\|K_{n}\right\|_{\mathrm{HS}}=0$, then 


$$
\lim _{n \rightarrow \infty}\left\|\left|A+K_{n}\right|-|A|\right\|_{\mathrm{HS}}=0
$$

for any bounded linear operator $A$.

For this purpose, we shall prove a weaker version of Theorem 1 where the coefficient $2^{1 / 2}$ is replaced by a larger number. (It then proves the above Proposition if we set $B=A+K_{n}$.)

Lemma 1. Let $A$ be a bounded positive definite selfadjoint operator and $K$ be in the Hilbert Schmidt class, both acting on a separable Hilbert space $\mathbf{H}$. Then there exists a selfadjoint operator $C$ in the Hilbert-Schmidt class such that

$$
\begin{aligned}
& A C+C A=K^{*} A+A K, \\
&\|C-\operatorname{Re} K\|_{\mathrm{HS}} \leqq\|\operatorname{Im} K\|_{\mathrm{HS}}, \\
&\|C\|_{\mathrm{HS}} \leqq 2^{1 / 2}\|K\|_{\mathrm{HS}}, \\
&\left\|C^{2}-K^{*} K\right\|_{\mathrm{tr}} \leqq\left(2+2^{1 / 2}\right)\|K\|_{\mathrm{HS}}\|\operatorname{Im} K\|_{\mathrm{HS}},
\end{aligned}
$$

where $2 \operatorname{Re} K=K+K^{*}, 2 i \operatorname{Im} K=K-K^{*}$.

Proof. We first prove the case where $A$ has a pure point spectrum. Let $A=\sum_{i=1}^{\infty} \lambda_{i} E_{i}$ with $E_{i}^{*}=E_{i}=E_{i}^{*}, \operatorname{dim} E_{i}=1$ (degeneracy of $\lambda$ 's allowed), $E_{i} \perp E_{j}(i \neq j)$ and $\lambda_{j}>0$. Let $\Psi_{i} \in E_{i} H$ and $\left\|\Psi_{i}\right\|=1$. Let $K_{i j}=\left(K \Psi_{j}, \Psi_{i}\right)$ and

$$
\begin{aligned}
C_{i j} & =\left(\lambda_{i}+\lambda_{j}\right)^{-1}\left(\lambda_{i} K_{i j}+\lambda_{j} \overline{K_{j i}}\right) \\
& =(\operatorname{Re} K)_{i j}+i\left(\lambda_{i}+\lambda_{j}\right)^{-1}\left(\lambda_{i}-\lambda_{j}\right)(\operatorname{Im} K)_{i j},
\end{aligned}
$$

where $\bar{K}_{i j}$ is the complex conjugate of $K_{i j},(\operatorname{Re} K)_{i j}=(1 / 2)\left(K_{i j}+\bar{K}_{j i}\right)$ and $(\operatorname{Im} K)_{i j}=(1 / 2 i)\left(K_{i j}-\bar{K}_{j i}\right)$. We then obtain $C_{i j}=\overline{C_{j i}}$ and

$$
\begin{aligned}
\sum_{i j}\left|C_{i j}-(\operatorname{Re} K)_{i j}\right|^{2} & =\sum_{i j}\left|\left(\lambda_{i}+\lambda_{j}\right)^{-1}\left(\lambda_{i}-\lambda_{j}\right)(\operatorname{Im} K)_{i j}\right|^{2} \\
& \leqq \sum_{i j}\left|(\operatorname{Im} K)_{i j}\right|^{2} .
\end{aligned}
$$

Hence, there exists a selfadjoint operator $C$ in the Hilbert-Schmidt class such that $C_{i j}=\left(C \Psi_{j}, \Psi_{i}\right)$ and (4.2) is satisfied. (Because $A$ is assumed to be positive definite, $\Sigma E_{i}=1$.) Then we obtain (4.3) by the following computation

$$
\begin{aligned}
\|C\|_{\mathrm{HS}} & \leqq\|C-\operatorname{Re} K\|_{\mathrm{HS}}+\|\operatorname{Re} K\|_{\mathrm{HS}} \\
& \leqq 2^{1 / 2}\left(\|\operatorname{Im} K\|_{\mathrm{HS}}^{2}+\|\operatorname{Re} K\|_{\mathrm{HS}}^{2}\right)^{1 / 2} \\
& =2^{1 / 2}\|K\|_{\mathrm{HS}} .
\end{aligned}
$$

From (4.5), (4.1) is checked for $\Psi=\Psi_{i}$ and $\Phi=\Psi_{j}$ and hence for all $\Phi$ and $\Psi$.

To prove (4.4), we use the following inequality:

$$
\begin{aligned}
\left\|C^{2}-K^{*} K\right\|_{\mathrm{tr}} & =(1 / 2)\left\|\left(C-K^{*}\right)(C+K)+\left(C+K^{*}\right)(C-K)\right\|_{\mathrm{tr}} \\
& \leqq\|C-K\|_{\mathrm{HS}}\|C+K\|_{\mathrm{HS}} .
\end{aligned}
$$


In addition, we have

$$
\begin{aligned}
& \|C-K\|_{\mathrm{HS}}^{2}=\|C-\operatorname{Re} K\|_{\mathrm{HS}}^{2}+\|\operatorname{Im} K\|_{\mathrm{HS}}^{2} \leqq 2\|\operatorname{Im} K\|_{\mathrm{HS}}^{2}, \\
& \|C+K\|_{\mathrm{HS}} \leqq\|C\|_{\mathrm{HS}}+\|K\|_{\mathrm{HS}} \leqq\left(1+2^{1 / 2}\right)\|K\|_{\mathrm{HS}} .
\end{aligned}
$$

Therefore we obtain (4.4). [Actually, $\left(2+2^{1 / 2}\right)$ in (4.4) can be easily improved to $\left.\left(1+5^{1 / 2}\right) \cdot\right]$

We now consider the general case. By a result of von Neumann [3], there exist for any $\varepsilon>0$ a selfadjoint operator $L_{\varepsilon}$ in the Hilbert-Schmidt class and a selfadjoint operator $B_{\varepsilon}$ with a pure point spectrum such that $A=B_{\varepsilon}+L_{\varepsilon}-2 \varepsilon$ and $\left\|L_{\varepsilon}\right\|_{\mathrm{HS}}<\varepsilon$. For this $B_{\varepsilon}$ and $K$, let $C_{\varepsilon}$ be the operator $C$ constructed above. Then $\left\|C_{\varepsilon}\right\|_{\mathrm{HS}} \leqq 2^{1 / 2}\|K\|_{\mathrm{HS}}$ and $B_{\varepsilon} \geqq \varepsilon$. Hence there exists a weak accumulation point $C$ of $C_{\varepsilon}$ as $\varepsilon \rightarrow 0$ in the Hilbert space of the Hilbert-Schmidt operators (with the inner product $\left\langle C_{1}, C_{2}\right\rangle=\operatorname{tr} C_{2}^{*} C_{1}$ ). Due to the reality of $\operatorname{tr} D C$ for all $D=D^{*}, C$ is selfadjoint. Since

$$
\left(B_{\varepsilon} \Phi, K \Psi\right)+\left(K \Phi, B_{\varepsilon} \Psi\right)=\left(C_{\varepsilon} \Phi, B_{\varepsilon} \Psi\right)+\left(B_{\varepsilon} \Phi, C_{\varepsilon} \Psi\right)
$$

holds for all $\Phi$ and $\Psi$, and since $B_{\varepsilon} \Phi$ and $B_{\varepsilon} \Psi$ tend strongly to $A \Phi$ and $A \Psi$, respectively, we obtain the same relation for $A, K$ and $C$, which shows (4.1). From (4.2), (4.3) and the estimate (4.4) for $C_{\varepsilon}$, the same inequalities hold for the weak accumulation point $C$ and hence (4.2), (4.3) and (4.4) holds for this $C$.

Remark. Suppose that $\operatorname{ker} A=0$. From (4.1), it follows that

$$
C=\operatorname{Re} K+i \int\left(\lambda+\lambda^{\prime}\right)^{-1}\left(\lambda-\lambda^{\prime}\right) d E(\lambda)(\operatorname{Im} K) d E\left(\lambda^{\prime}\right),
$$

where $A=\int \lambda d E(\lambda)$. Since $C$ is uniquely determined, $C_{\varepsilon}$ actually converges to $C$ in this case.

Other inequalities we need are the following: For $A^{*}=A$ and $B^{*}=B$,

$$
\||A|-|B|\|_{\mathrm{HS}} \leqq\|A-B\|_{\mathrm{HS}} .
$$

This is given in Lemma 5.2, [1]. For positive selfadjoint $A$ and $B$,

$$
\|A-B\|_{\mathrm{HS}}^{2} \leqq\left\|A^{2}-B^{2}\right\|_{\mathrm{tr}} .
$$

This is given in Lemma 4.1, [4].

We now prove a weaker version of Theorem 1. First consider the case where $A$ is positive. Let $K=B-A$. If $K$ is not in the Hilbert-Schmidt class, the inequality holds for the trivial reason that the right hand side is $+\infty$. If $K$ is in the Hilbert-Schmidt class, then use $C$ given in Lemma 1. We obtain

$$
|B|=\left((A+K)^{*}(A+K)\right)^{1 / 2}=\left\{(A+C)^{2}+\left(K^{*} K-C^{2}\right)\right\}^{1 / 2} .
$$

Hence by (4.9) and (4.10) we obtain

$$
\begin{aligned}
\||B|-A\|_{\mathrm{HS}} & \leqq\||B|-|A+C|\|_{\mathrm{HS}}+\||A+C|-A\|_{\mathrm{HS}} \\
& \leqq\left\|K^{*} K-C^{2}\right\|_{\mathrm{tr}}^{1 / 2}+\|C\|_{\mathrm{HS}} \\
& \leqq \beta\|K\|_{\mathrm{HS}}=\beta\|B-A\|_{\mathrm{HS}},
\end{aligned}
$$

where $\beta$ may be taken to be $2^{1 / 2}+\left(2+2^{1 / 2}\right)^{1 / 2}$. 
Now we consider the general case. Let $F$ be the projection operator on (Range $A)^{\perp}$. We assume that $B-A=K$ is in the Hilbert-Schmidt class. Then $F(B-A)=F B$ is in the Hilbert-Schmidt class with $\|F B\|_{\mathrm{HS}} \leqq\|B-A\|_{\mathrm{HS}}$.

Let $A=U|A|$ be the polar decomposition of $A$. We have $U U^{*}=1-F$. Then

$$
\begin{aligned}
\||B|-|A|\|_{\mathrm{HS}} & \leqq\||B|-|(1-F) B|\|_{\mathrm{HS}}+\left\|\left|U^{*} B\right|-|A|\right\|_{\mathrm{HS}} \\
& \leqq\|F B\|_{\mathrm{HS}}+\beta\left\|U^{*}(B-U|A|)\right\|_{\mathrm{HS}} \\
& \leqq(\beta+1)\|B-A\|_{\mathrm{HS}},
\end{aligned}
$$

where we have used (4.10), $|B|^{2}-|(1-F) B|^{2}=|F B|^{2}$ and (4.11).

\section{Proof of Theorem 1}

Lemma 2. If $K$ is a Hilbert-Schmidt class operator and a sequence of bounded linear operators $Q_{n}$ tends to $Q$ strongly, then

$$
\lim _{n}\left\|\left(Q_{n}-Q\right) K\right\|_{\mathrm{HS}}=0, \quad \lim _{n}\left\|K\left(Q_{n}^{*}-Q^{*}\right)\right\|_{\mathrm{HS}}=0 .
$$

Proof. Since the sequence $Q_{n}$ has a strong limit, sup $\left\|Q_{n}\right\| \equiv q$ is finite. For any $\varepsilon>0$, there exists a finite rank operator $K_{\varepsilon}$ such that $\left\|K-K_{\varepsilon}\right\|_{H S}<\varepsilon$. Since the range of $K_{\varepsilon}$ has a finite dimension, there exists $N_{\varepsilon}$ such that for $n>N_{\varepsilon}$

$$
\|\left(Q_{n}-Q\right) \mid \text { Range } K_{\varepsilon} \|<\varepsilon \text {. }
$$

Then for $n>N_{\varepsilon}$, we obtain

$$
\begin{aligned}
\left\|\left(Q_{n}-Q\right) K\right\|_{\mathrm{HS}} & \leqq\left\|\left(Q_{n}-Q\right)\left(K-K_{\varepsilon}\right)\right\|_{\mathrm{HS}}+\left\|\left(Q_{n}-Q\right) K_{\varepsilon}\right\|_{\mathrm{HS}} \\
& \leqq\left\|Q_{n}-Q\right\|\left\|K-K_{\varepsilon}\right\|_{\mathrm{HS}}+\|\left(Q_{n}-Q\right) \mid \text { Range } K_{\varepsilon}\|\| K_{\varepsilon} \|_{\mathrm{HS}} \\
& \leqq(q+\|Q\|+\|K\|+\varepsilon) \varepsilon .
\end{aligned}
$$

This proves the first relation in Lemma 2. The second follows from the first by $\left\|K\left(Q_{n}^{*}-Q^{*}\right)\right\|_{\mathrm{HS}}=\left\|\left(Q_{n}-Q\right) K^{*}\right\|_{\mathrm{HS}}$.

Proof of Theorem 1. We first consider the case where $A \geqq 0$ and $A$ has a pure point spectrum and hence $A=\sum_{i=1}^{\infty} \lambda_{i} E_{i}$ with $E_{i} \perp E_{j}(i \neq j)$ and $\operatorname{dim} E_{i}=1$. Let $B$ $=A+K$ with $\|K\|_{\mathrm{HS}}<\infty$. Let $F_{n}=\sum_{i=1}^{n} E_{i}$. By the special case of Theorem 1 proved in Sect. 2, we obtain

$$
\begin{aligned}
\left\|\left|A+F_{n} K F_{n}\right|-A\right\|_{\mathrm{HS}} & =\left\|\left|F_{n} A+F_{n} K F_{n}\right|-F_{n} A\right\|_{\mathrm{HS}} \\
& \leqq 2^{1 / 2}\left\|F_{n} K F_{n}\right\|_{\mathrm{HS}} .
\end{aligned}
$$

Since $F_{n} K F_{n}-K=\left(F_{n} K-K\right)+F_{n}\left(K F_{n}-K\right)$ and $\lim F_{n}=1$, we have

$$
\lim \left\|F_{n} K F_{n}-K\right\|_{\mathrm{HS}}=0
$$

by Lemma 2. By Proposition, we have

$$
\lim \left\|\left|A+F_{n} K F_{n}\right|-|A+K|\right\|_{\mathrm{HS}}=0 .
$$


Hence by the same proposition,

$$
\||A+K|-A\|_{\mathrm{HS}} \leqq 2^{1 / 2} \sup _{n}\left\|F_{n} K F_{n}\right\|_{\mathrm{HS}}=2^{1 / 2}\|K\|_{\mathrm{HS}} .
$$

Next, we consider the case where $A \geqq 0$ but $A$ may have an arbitrary (bounded) spectrum. By the von Neumann approximation theorem and Proposition, (5.1) for this case follows from (5.1) for a positive $A$ with a pure point spectrum.

Note that if $A \geqq 0, A=B_{\varepsilon}+K_{\varepsilon}, B_{\varepsilon}^{*}=B_{\varepsilon}$ and $F$ is the spectral projection of $B_{\varepsilon}$ for $(-\infty, 0), \quad$ then $0 \leqq-B_{\varepsilon} F=F K_{\varepsilon} F-F A F \leqq F K_{\varepsilon} F, \quad-B_{\varepsilon} F$ $=\left(K F_{\varepsilon} F\right)^{1 / 2} Q\left(K F_{\varepsilon} K\right)^{1 / 2}$ for $\|Q\| \leqq 1,\left\|B_{\varepsilon} F\right\|_{\mathrm{HS}} \leqq\left\|K_{\varepsilon}\right\|_{\mathrm{HS}}$ and hence we may assume $B_{\varepsilon} \geqq 0$ by including $F B_{\varepsilon} F$ in $K_{\varepsilon}$.

If Range $A=H$, then the method of proof at the end of the preceding section works with $F=0$ and (1.1), for such a case follows from (1.1) for the case of a positive $A$ just proved.

Finally we consider a general case. Let $u$ be an isometry with $1-u u^{*}$ having an infinite dimension. Since $\left|B u^{*}\right|^{2}=u|B|^{2} u^{*}$ and $\left|A u^{*}\right|^{2}=u|A|^{2} u^{*}$, we have

$$
\left\|\left|B u^{*}\right|-\left|A u^{*}\right|\right\|_{\mathrm{HS}}=\left\|u(|B|-|A|) u^{*}\right\|_{\mathrm{HS}}=\||B|-|A|\|_{\mathrm{HS}}
$$

where we have used $u^{*} u=1$. In the same way

$$
\left\|B u^{*}-A u^{*}\right\|_{\mathrm{HS}}=\|B-A\|_{\mathrm{HS}} .
$$

Let $v$ be a partial isometry such that $v^{*} v \leqq 1-u u^{*}$ and Range $v$ $=\left(\text { Range } A u^{*}\right)^{\perp}$. Let $L_{\varepsilon}$ be an operator such that $L_{\varepsilon}=L_{\varepsilon}^{*}$, Range $L_{\varepsilon}=$ Range $v$ and $\left\|L_{\varepsilon}\right\|_{\mathrm{HS}}<\varepsilon$ for $\varepsilon>0$. Let $A_{\varepsilon}=A u^{*}+L_{\varepsilon} v$. Then Range $A_{\varepsilon}=H$, and hence we may use (1.1) for the pair $B u^{*}$ and $A_{\varepsilon}$ (instead of $B$ and $A$ ) to obtain

$$
\left\|\left|B u^{*}\right|-\left|A_{\varepsilon}\right|\right\|_{\mathrm{HS}} \leqq 2^{1 / 2}\left\|B u^{*}-A_{\varepsilon}\right\|_{\mathrm{HS}} .
$$

As $\varepsilon \rightarrow 0$, we obtain $\left\|A_{\varepsilon}-A u^{*}\right\|_{\mathrm{HS}} \rightarrow 0$. By Proposition, (5.4) implies

$$
\left\|\left|B u^{*}\right|-\left|A u^{*}\right|\right\|_{\mathrm{HS}} \leqq 2^{1 / 2}\left\|B u^{*}-A u^{*}\right\|_{\mathrm{HS}} .
$$

By (5.2) and (5.3), we obtain (1.1) for the general case.

Acknowledgement. The authors would like to thank Professor T. Ando for critical reading of the manuscript.

Note added in proof. A. Kishimoto kindly pointed out the following simplification of the proof in Sects. 4 and 5: It is easy to prove that if $T_{n} \rightarrow T$ strongly, then $\|T\|_{\text {H.s. }} \leqq \underline{\lim }\left\|T_{n}\right\|_{\text {H.S. }}$. If $B=B_{\varepsilon}+L$ with $\|L\|_{\text {H.S }}<\infty$ and a pure point spectrum for $B_{\varepsilon}$ and $K=A-B$ is in the H.S. class, then

$$
\left\|\left|B_{\varepsilon}+E_{n}(L+K) E_{n}\right|-\left|B_{\varepsilon}+E_{n} L E_{n}\right|\right\|_{\text {H.S. }} \leqq \sqrt{2}\|K\|_{\text {H.S }}
$$

and the strong limit of the operator difference is $|A|-|B|$. Hence the desired conclusion follows. 


\section{References}

1. Araki, H.: Publ. RIMS Kyoto Univ. 6, 385-442 (1971)

2. Araki, H.: Pac. J. Math. 50, 309-354 (1974)

3. von Neumann, J.: Actual. Sci. Ind. 299, (1935)

4. Powers, R.T. and Størmer, E.: Commun. Math. Phys. 16, 1-33 (1970)

Communicated by H. Araki

Received March 27, 1981 